\title{
Demosaicked Image PostProcessing Framework
}

\author{
Rastislav Lukac, Karl Martin and Konstantinos N. Plataniotis \\ Bell Canada Multimedia Laboratory, The Edward S. Rogers Sr. Department of ECE, \\ University of Toronto, 10 King's College Road, Toronto, M5S 3G4 Ontario, Canada \\ lukacr@ieee.org, kmartin@dsp.utoronto.ca, kostas@dsp.utoronto.ca
}

\begin{abstract}
A new demosaicked image postprocessing method for single-sensor imaging devices is introduced. The method is capable of removing visual demosaicking artifacts in the restored, full color images obtained by cost-effective color filter array interpolators. The utilization of the normalized color-ratio model and the original underlying Bayer pattern structure allows for the removal of false colors and eliminates color shifts introduced during demosaicking. At the same time, it yields excellent improvements in terms of objective image quality measures. Moreover, the computational simplicity of the method suggests that it can be effectively implemented either in hardware or software.
\end{abstract}

Keywords: Single-sensor imaging, color filter array interpolation, Bayer pattern, demosaicking, demosaicked image postprocessing, visual artifacts.

\section{INTRODUCTION}

Color filter array interpolation (demosaicking) is an essential element in single-sensor imaging devices such as digital cameras, and mobile telephones and personal digital assistants (PDAs) with embedded image capturing capabilities. Most of these devices use a single charged couple device (CCD) or a complementary metal oxide semiconductor (CMOS) sensor with a color filter array (CFA). Using the most popular Bayer CFA pattern [1], the sensor produces a two-dimensional array with each spatial location containing only a red $(R)$, green $(G)$, or blue (B) component. The restored color image output is obtained by interpolating the missing color components from the spatially adjacent CFA data [2],[7],[8],[9].

Due to constraints on cost-effective solutions, the demosaicked images are lacking in sharpness and contain false colors [9]. To remove color artifacts present in the demosaicked images and provide the end-user with visually pleasing color output, the proposed method utilizes a normalized color-ratio model and the underlying Bayer pattern structure. Correcting the previously interpolated color components, the method impressively improves the quality of the color output produced by well-known CFA interpolation schemes. The postprocessing framework removes false color artifacts, increases sharpness, and never decreases image fidelity. At the same time, it also yields excellent improvements in terms of objective image quality measures.

\section{PROPOSED METHOD}

Let us consider, a $K_{1} \times K_{2}$ full color image $\mathrm{x}: Z^{2} \rightarrow Z^{3}$ representing a two-dimensional matrix of three-component RGB color samples $\mathbf{x}_{(p, q)}=\left[x_{(p, q) 1}, x_{(p, q) 2}, x_{(p, q) 3}\right]$, with $x_{(p, q) k}$ denoting the $\mathrm{R}$ component for $k=1$, the $\mathrm{G}$ component for $k=2$ and the $\mathrm{B}$ component for $k=3$. The coordinates $p=1,2, \ldots, K_{1}$ and $q=1,2, \ldots, K_{2}$ denote the spatial position of a pixel in vertical (image rows) and horizontal (image columns) directions respectively. Assuming the GRGR-phased Bayer CFA pattern [1], the demosaicked color image $\mathbf{x}$ has been restored using the R CFA components $x_{(p, q) 1}$ located at (odd $p$, even $q$ ), the G CFA components $x_{(p, q) 2}$ located at (odd $p$, odd $q$ ) and (even $p$, even $q$ ), and the B CFA components $x_{(p, q) 3}$ located at (even $p$, odd $q$ ). Thus, each full color pixel $\mathbf{x}_{(p, q)}$ consists of a single original CFA component and two interpolated components. Since the original CFA data remain unchanged in the demosaicked images, they can be considered as the input to the postprocessor as well.

It is well-known that the sample density of the $\mathrm{G}$ component in many RGB color filter arrays (such as the Bayer pattern) is double that of the $\mathrm{R}$ and $\mathrm{B}$ components. This is mainly due to the fact that $\mathrm{G}$ components are considered to be better representative of the underlying luminance characteristics. It is therefore reasonable to start the postprocessing with the $\mathrm{G}$ components.

The postprocessing procedure first updates the estimated $\mathrm{G}$ components $x_{(p, q) 2}$ at the spatial locations corresponding to the original $\mathrm{R}$ or $\mathrm{B}$ components as follows [9]:

$$
x_{(p, q) 2}=-\beta+\left(x_{(p, q) k}+\beta\right) \operatorname{mean}_{(i, j) \in \zeta}\left\{\frac{x_{(i, j) 2}+\beta}{x_{(i, j) k}+\beta}\right\}
$$

where $\beta$ is a constant used to normalize color-ratios $\left(x_{(i, j) 2}+\beta\right) /\left(x_{(i, j) k}+\beta\right)$, with $k=1$ for an original $\mathrm{R}$ position (odd $p$, even $q$ ) or $k=3$ for an original B position (even $p$, odd $q$ ). The original $\mathrm{G}$ components $x_{(i, j) 2}$ are used in conjunction with the interpolated color components $x_{(i, j) k}$ to generate a normalized color-ratio description [9], 
defined here as $\mathrm{G} / \mathrm{R}$ (for $k=1$ ) or $\mathrm{G} / \mathrm{B}$ (for $k=3$ ) ratios over the neighborhood $\zeta=\{(p-1, q),(p, q-1),(p, q+$ 1), $(p+1, q)\}$.

It has been widely observed that in low-quality demosaicked images, unnatural transitions in hue correspond to false colors. The variations in hue are often more pronounced than expected in a natural scene due to the fact that neighboring pixels are used in the recovery of unknown values. In demosaicking, the changes in hue are commonly attributed to the changes in color-ratios [2],[3],[7]. Instead of the conventional color-ratio model of [3] which fails in high-frequency image areas, the proposed method normalizes the color-ratios and uses them to estimate the $\mathrm{G}$ component $x_{(p, q) 2}$ based on the original component $x_{(p, q) k}$. Moreover, the normalization (linear shifting) through $\beta$ avoids singularities in the color-ratio calculations [9].

The next step involves the correction of the CFAinterpolated inputs $x_{(p, q) k}$, for $\mathrm{R}(k=1)$ located at the original $\mathrm{B}$ positions, and $\mathrm{B}(k=3)$ located at the original R positions. The corrected $\mathrm{G}$ component $x_{(p, q) 2}$ of (1) is used to estimate the component $x_{(p, q) k}$ by utilizing the $\mathrm{R} / \mathrm{G}$ or $\mathrm{B} / \mathrm{G}$ LCRs as follows [9]:

$$
x_{(p, q) k}=-\beta+\left(x_{(p, q) 2}+\beta\right) \operatorname{mean}_{(i, j) \in \zeta}\left\{\frac{x_{(i, j) k}+\beta}{x_{(i, j) 2}+\beta}\right\}
$$

where $\zeta=\{(p-1, q-1),(p-1, q+1),(p+1, q-1),(p+$ $1, q+1)\}$. This operation is similar to (1) except that $\zeta$ is adjusted to take into account locations of the original $\mathbf{R}$ or B CFA data $x_{(i, j) k}$. The $x_{(i, j) 2}$ corresponds to the corrected $\mathrm{G}$ component from the previous step.

The postprocessing procedure is completed with the correction of the interpolated $\mathrm{R}$ and $\mathrm{B}$ inputs $x_{(p, q) k}$ located at spatial positions corresponding to the original G CFA data. Thus, the postprocessing step (2) is repeated, now utilizing the original $\mathrm{G}$ component $x_{(p, q) 2}$ and $\zeta=\{(p-1, q),(p, q-$ $1),(p, q+1),(p+1, q)\}$. Two of the values $x_{(i, j) k}$ of $(2)$ are original components and the other two are corrected components previously obtained using (2), whereas $x_{(i, j) 2}$ denotes the corrected $\mathrm{G}$ components of (1).

Since fine structural elements are not always wide enough to span the neighborhood used in interpolating the color components, the large dynamics of the CFA data inside $\zeta$ prevents the recovery of the color information at edges and the demosaicking schemes may introduce color artifacts into the restored image. Using a sufficiently large parameter $\beta$, the color-ratios of (1) and (2) are normalized into the similar values which significantly reduce failures of low-pass (averaging) operators. Thus, the accuracy of the postprocessor defined in (1) and (2) depends only on the multiplicative normalization factor $\left(x_{(p, q) k}+\beta\right)$ in (1) or $\left(x_{(p, q) 2}+\beta\right)$ in (2), and the subtractive normalization through $-\beta$. Since $x_{(p, q) k}$ of (1) and $x_{(p, q) 2}$ of (2) are the color components which contain high-frequency informa-
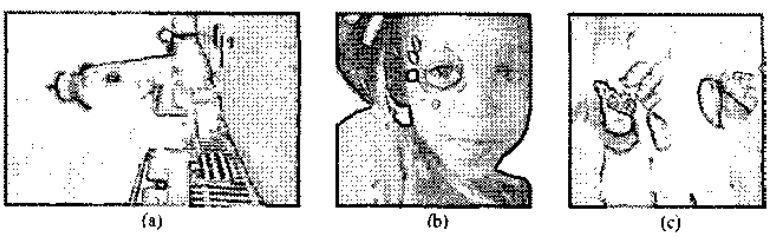

Fig. 1. Test set: (a) Lighthouse, (b) Kid, (c) Parrots.

tion at the interpolated locations, the postprocessing preserves the structural content of the image including fine details.

Operating on modified LCRs $\left(x_{(i, j) 2}+\beta\right) /\left(x_{(i, j) k}+\beta\right)$ or $\left(x_{(i, j) k}+\beta\right) /\left(x_{(i, j) 2}+\beta\right)$ with sufficiently large $\beta$ decreases the input's dynamic range inside the local neighborhood $\zeta$, thus regulating the smoothing process. Therefore, the proposed normalization boosts the performance of the imaging solution. Note that the method can be adapted to other CFAs by simply adjusting the local neighborhood $\zeta$ and the concept of the proposed regularization can also be applied to the demosaicking algorithms of [2],[3],[7], which utilize color-ratios.

\section{EXPERIMENTAL RESULTS}

A number of color images (Fig. I) with an 8-bit per channel representation have been used to evaluate the proposed postprocessing framework. The images $(768 \times 512$ in size for Lighthouse and $512 \times 512$ for others) were captured using three-sensor devices. Mosaic versions of the images are created using by a GRGR-phased Bayer CFA filter, following standard practices [7],[8],[9]. Demosaicked images are generated using well-known Bayer CFA interpolation algorithms such as saturation-based adaptive inverse gradient (SAIG) [2], constant hue-based interpolation (CHI) [3], median filter interpolation (MFI) [4], edge-adaptive color interpolation (ECI) [5], principle vector method (PVM) [6], Kimmel's algorithm (KA) [7], and bilinear interpolation (BI) [8]. The results are objectively evaluated through the mean square error (MSE) and the normalized color difference criterion (NCD) [10].

Fig. 2 illustrates the influence of $\beta$ in the proposed postprocessor. As it is also shown in Fig. 3, the performance of the proposed method increases with $\beta$. Through the proposed normalization the method eliminates color shifts observed in high-frequency image transitions and produces true colors. Note that all subsequent results have been obtained using $\beta=512$ which is equivalent to double the maximum dynamic range of the color components.

Tables 1 -3 summarize the objective measurements comparing the original demosaicking results and the postprocessed results, corresponding to the test images shown in 

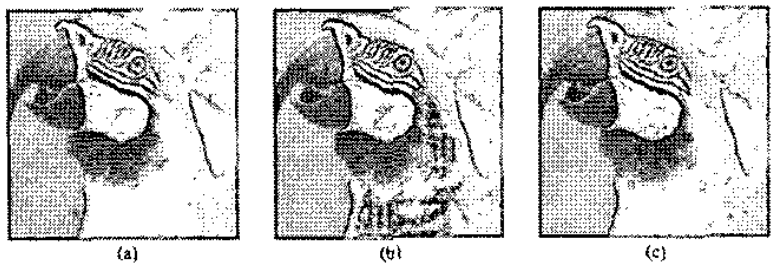

Fig. 2. Zoomed part of the test image Parrots: (a) BI-CFA output, (b-c) the proposed postprocessing method output: (b) $\beta=0$, (c) $\beta=10$.
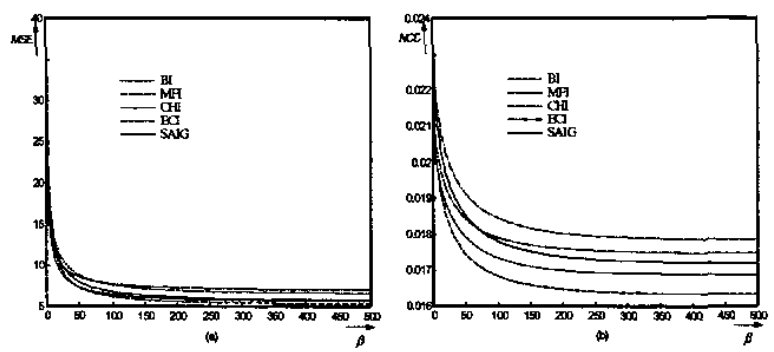

Fig. 3. Performance of the proposed postprocessing method depending on $\beta$ : (a) MSE, (b) NCD.

Fig.I. In all cases, the postprocessing operation provides significant improvement. The most significant improvement can be found with the postprocessing of the BI generated images.

Figs. 4-6 show zoomed-in portions of the test images in areas that are typically problematic for CFA-based interpolation schemes. False color distortion is quite evident for BI, CHI, ECI and SAIG. Upon applying the proposed procedure, the effect is largely removed. In conjunction with the removal of false colors, an increase in image sharpness is observed. As it can be seen, the postprocessor was able to cancel the distortion effects and produced an image with no impairments or visual artifacts.

Table 1. Results related to the image Lighthouse.

\begin{tabular}{crrrr}
\hline Type & \multicolumn{2}{c}{ demosaicked } & \multicolumn{2}{c}{ postprocessed } \\
\hline Method & MSE & NCD & MSE & NCD \\
\hline BI & 105.8 & 0.0653 & 18.0 & 0.0305 \\
MFI & 31.0 & 0.0380 & 12.9 & 0.0261 \\
CHI & 56.1 & 0.0501 & 19.6 & 0.0307 \\
ECI & 22.6 & 0.0370 & 8.5 & 0.0242 \\
SAIG & 23.9 & 0.0403 & 9.8 & 0.0293 \\
KA & 19.0 & 0.0365 & 10.1 & 0.0303 \\
PVM & 23.1 & 0.0366 & 8.7 & 0.0249 \\
\hline
\end{tabular}

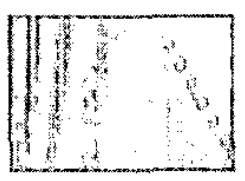

(a)
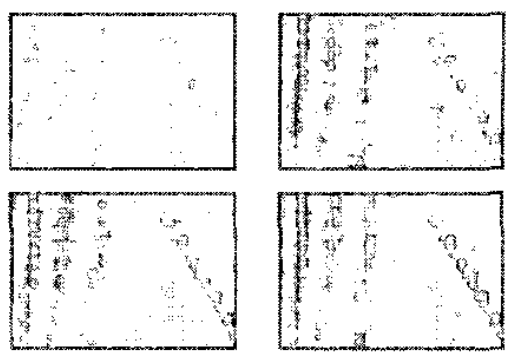

(b)
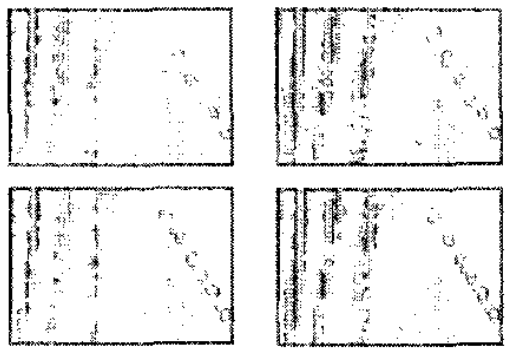

(d)
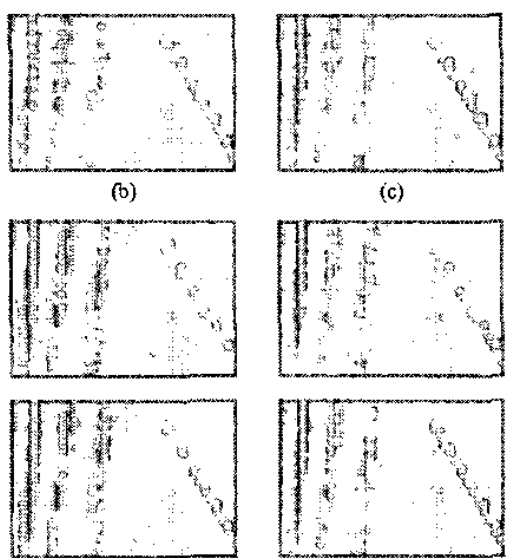

(c)
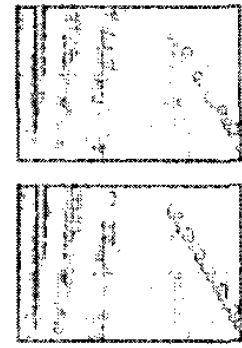

Fig. 4. Enlarged parts of the Lighthouse image suffering from aliasing: (a) the original image, (b)-(f) the restored outputs before (top line) and after (bottom line) postprocessing: (b) $\mathrm{BI}$, (c) ECI, (d) KA, (e) PVM, (f) SAIG.

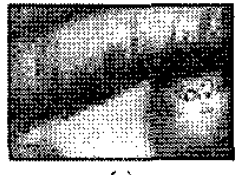

(a)
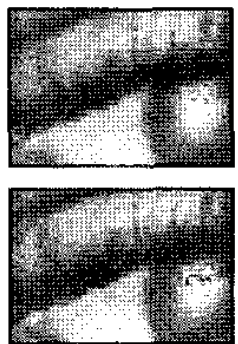

(c)
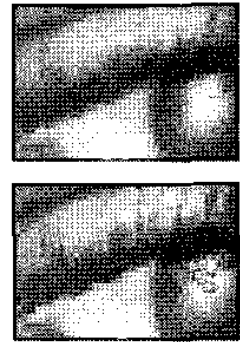

(b)
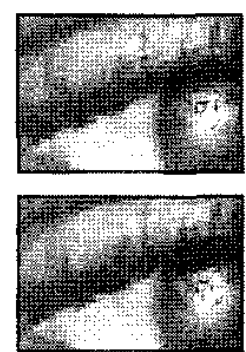

(e)
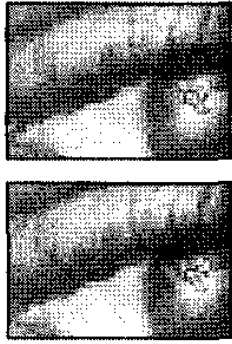

(c)
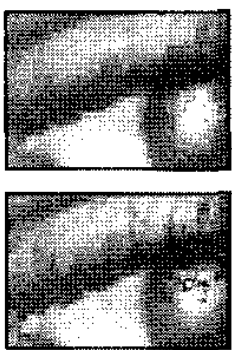

(f)

Fig. 5. Enlarged parts of the original Kid image (a), and the restored outputs (b)-(f) before (top line) and after (bottom line) postprocessing: (b) BI, (c) MFI, (d) $\mathrm{CHI},(e) \mathrm{ECl}$, (f) SAIG. 


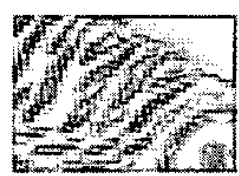

(a)

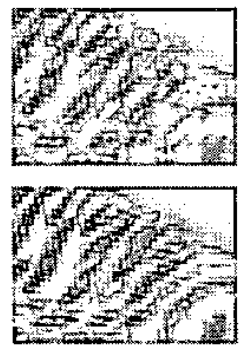

(d)
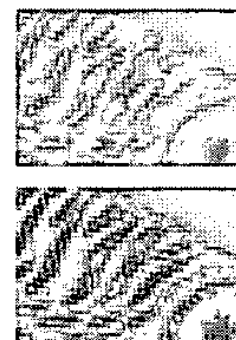

(b)

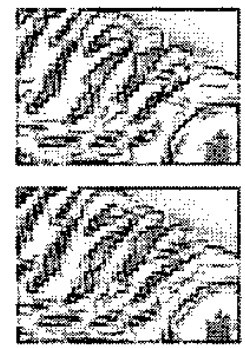

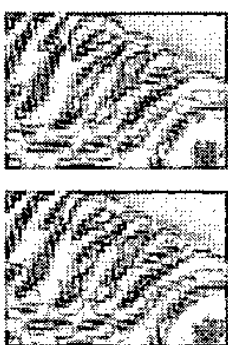

(c)
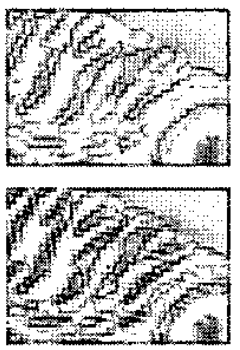

(f)

Fig. 6. Enlarged parts of the original Parrots image (a), and the restored outputs (b)-(f) before (top line) and after (bottom line) postprocessing: (b) BI, (c) MFI, (d) $\mathrm{CHI},(e) \mathrm{ECl}$, (f) SAIG.

\section{CONCLUSION}

A new technique for demosaicked image postprocessing was introduced. The underlying Bayer structure included in the restored image is utilized along with a normalized colorratio model. For all test images, the scheme reduced or completely removed false color artifacts while increasing image sharpness. Combined with any demosaicking scheme, the proposed method can be seen as a correction of the CFA interpolation algorithm.

\section{References}

[1] B.E. Bayer, "Color imaging array," U.S. Patent 3971 065, 1976.

[2] C. Cai, T.H. Yu, and S.K. Mitra, "Saturation-based adaptive inverse gradient interpolation for Bayer pattern images," IEE Proceedings - Vision, Image, Signal Processing, vol. 148, pp. 202-208, June 2001.

[3] D.R. Cok, "Signal processing method and apparatus for producing interpolated chrominance values in a sampled color image signal," US Patent 4642 678, 1987.

[4] W.T. Freeman, "Median filter for reconstructing missing color samples," U.S. Patent 5373 322, 1988.

[5] B.S. Hur and M.G. Kang, "High definition color interpolation scheme for progressive scan CCD image sensor," IEEE Transactions on Consumer Electronics, vol. 47, pp. 179-186, Feb. 2001.

[6] R. Kakarala, Z. Baharav, "Adaptive demosaicing with the principle vector method," IEEE Transactions on Consumer Electronics, vol. 48, pp. 932-937, Nov. 2002.

[7] R. Kimmel, "Demosaicing: Image reconstruction from color CCD samples," IEEE Transactions on Image Processing, vol. 8, pp. 1221-1228, September 1999.

[8] P. Longere, X. Zhang, P.B. Delahunt, and D.H. Brainard, "Perceptual assessment of demosaicing algorithm performance," Proceedings of the IEEE, vol. 90, pp. 123-132, Jan. 2002.

[9] R. Lukac, K. Martin, K.N. Plataniotis, "Demosaicked image postprocessing using local color ratios," IEEE Transactions on Circuit and Systems for Video Technology, vol. 14, to appear, 2004.

[10] K.N. Plataniotis, A.N. Venetsanopoulos, Color Image Processing and Applications. Springer Verlag, 2000.

Table 2. Results related to the image Kid.

\begin{tabular}{crccc}
\hline Type & \multicolumn{2}{c}{ demosaicked } & \multicolumn{2}{c}{ postprocessed } \\
\hline Method & MSE & NCD & MSE & NCD \\
\hline BI & 42.6 & 0.0697 & 11.6 & 0.0421 \\
MFI & 12.7 & 0.0451 & 10.4 & 0.0395 \\
CHI & 37.3 & 0.0674 & 12.7 & 0.0461 \\
ECI & 34.5 & 0.0612 & 13.5 & 0.0458 \\
SAIG & 34.5 & 0.0620 & 15.8 & 0.0611 \\
KA & 37.3 & 0.0709 & 21.0 & 0.0696 \\
PVM & 25.9 & 0.0559 & 12.1 & 0.0423 \\
\hline
\end{tabular}

Table 3. Results related to the image Parrots.

\begin{tabular}{crrrr}
\hline Type & \multicolumn{2}{c}{ demosaicked } & \multicolumn{2}{c}{ postprocessed } \\
\hline Method & MSE & NCD & MSE & NCD \\
\hline BI & 29.3 & 0.0262 & 5.7 & 0.0169 \\
MFI & 6.8 & 0.0172 & 5.3 & 0.0163 \\
CHI & 16.7 & 0.0223 & 7.0 & 0.0174 \\
ECI & 16.2 & 0.0207 & 6.6 & 0.0178 \\
SAIG & 11.6 & 0.0224 & 8.2 & 0.0223 \\
KA & 91.9 & 0.0357 & 17.1 & 0.0268 \\
PVM & 11.1 & 0.0199 & 5.6 & 0.0169 \\
\hline
\end{tabular}

